\title{
LA IMPORTANCIA DE LOS BIOHUERTOS ESCOLARES COMO ESTRATEGIA DE APRENDIZAJE EN MATEMÁTICAS EN LA REGIÓN HUÁNUCO
}

\author{
Braulia TACUCHE ALVARADO ${ }^{1}$
}

\begin{tabular}{|lcc|}
\hline Recibido & $:$ & 20.02 .2020 \\
Aceptado & $:$ & 02.05 .2020 \\
Publicado & $:$ & 06.07 .2020 \\
\hline
\end{tabular}

RESUMEN: Las estrategias educativas que existen en la actualidad son diversos y se ajustan a la realidad de la población, región, situación económica e incluso al nivel de aprendizaje requerido. En el Perú, en los cuales sus principales deficiencias son el sector educativo, muchos de los docentes establecen metodologías para lograr que sus estudiantes, para los fines de esta investigación, puedan aplicar la resolución correcta de los problemas matemáticos, es por ello que luego de probar diversas estrategias, se ha logrado comprender que una acción sencilla y que no es complicada de realizar, para mejorar la capacidad de recepción y resolución de problemas aritméticos de enunciados verbales (PAEV) por parte de los estudiantes en esta región, es con la implementación de un biohuerto como parte de un proyecto estudiantil. Este artículo se enfocará en demostrar la relevancia de estos biohuertos en la enseñanza de la resolución de problemas aritméticos de enunciados verbales en los estudiantes de la región Huánuco.

Palabras claves: Biohuerto, aprendizaje, estrategia, resolución de problemas aritméticos de enunciados verbales-PAEV, Huánuco, proyecto.

The importance of school biogardens as a strategy of learning mathematics in the Huánuco region.

ABSTRACT: The educational strategies that currently exist are diverse and adapted to the reality of the population, region, economic situation and even the level of learning required. In Peru, in which the main deficiencies are the education sector, many of the teachers establish methodologies to ensure that their students, for the purposes of this research, can apply the correct solution to mathematical problems, that is why after trying different strategies, it has been possible to understand that a simple and not complicated action to improve the ability of students in this region to receive and solve arithmetic problems of verbal statements (PAEV), is with the implementation of a biogarden as part of a student project. This article will focus on demonstrating the relevance of these biogardens in teaching the solution of arithmetic problems of verbal statements in students of the Huanuco region.

Keywords: Bioorchard, learning, strategy, solving arithmetic problems of verbal statements-PAEV, Huanuco, project.

\footnotetext{
${ }^{1}$ Universidad Nacional Mayor de San Marcos. Email: bratacualva@ hotmail.com
} 


\section{Journal of the Academy $|98|$}

\section{INTRODUCCIÓN}

La educación es un aspecto fundamental, no solo para el desarrollo cognitivo, sino también evoca a un desarrollo emocional, social, en el cual se comprometa no solo estudiante, sino también el padre de familia y los maestros. Para ello los docentes, aprenden a identificar los principales problemas que pueden afectar a su población e impactar en la enseñanza de sus estudiantes.

En la región de Huánuco, con aproximadamente con una población de 847 mil habitantes (INEI, 2018), y con una población escolar de aproxima 89500 estudiantes entre educación básica y regular. Sin embargo, presenta muchos vacíos para la definición de estrategias de aprendizaje, además la escasa participación del Estado para proyectar nuevas alternativas pedagógicas impacta en la formación de estos estudiantes. Los proyectos de infraestructura educativa como consecuencia de la intervención del Estado, se encuentran parametrizadas en forma burocrática con plazos extensos que son innecesarios e injustos, ya que se demora más el recorrido del proyecto y sus observaciones en PRONIED (Programa Nacional de Infraestructura Educativa), que sea viable, rápida y óptima de su ejecución, en especial en la región sierra del Perú. (Avalos, 2019, 2)

Rojas, A., Salinas, L. y Ch, J. (2019) señalaban que el clima institucional fue un factor predominante en la carencia de un buen desarrollo de la gestión pedagógica de los docentes de las instituciones educativas de la región de Huánuco, dejando atrás su relevada importancia estratégica y de esa manera conllevando a una improvisación curricular. (pág. 3)

Es por ello, que en la actualidad parte de la identificación de los problemas no solo en Huánuco sino a nivel nacional es la escaza preparación tanto en matemáticas como en lenguajes, y dentro de esos dos aspectos es puntualmente el caso de las matemáticas subyugado a la dificultad de los estudiantes de aplicar estrategias para la resolución de Problemas Aritméticos de Enunciados Verbales o también llamadas PAEV, la cual forma parte de la investigación realizada.

Si bien, se han identificados varios aspectos que generan dificultades para la resolución de los PAEV; como, por ejemplo, el poco dominio de nuevos enfoques matemáticos, el escaso interés en investigación, pero también se identificó un aspecto interesante; la alimentación. De acuerdo a la zona geográfica de la región Huánuco que en un alto porcentaje está conformado por una basta selva, la alimentación principal se concentra a base de yuca, plátano, maíz, pituca y otros a escala menor, lo que, sin embargo, de acuerdo a las dietas balanceadas sugeridas por diversos nutricionistas esto no sería suficiente. El tener un biohuerto abre posibilidades de cultivar algunas otras verduras o frutas que aporten una buena cantidad de vitaminas, minerales fortaleciendo así nuestro organismo.

Parte del problema formulado recae en descubrir en si los biohuertos realmente aportan a la estrategia de aprender matemáticas en la resolución de los PAEV. Esto se justifica en las necesidades de buscar nuevas alternativas para la mejora de la calidad de enseñanza en los estudiantes huanuqueños, así como el llamado a la reflexión de valorar, pero desechar enfoques tradicionales que puedan no ser suficientes para la enseñanza y que fuera reflejado en la última Evaluación Censal de Estudiantes - 2017.

Para la presente investigación se han rescatado diversos datos de algunas entidades educativas que nos permitan conocer si realmente esta experiencia de crear biohuertos como proyecto para el mejoramiento del aprendizaje en matemática es factible o no.

\section{DESARROLLO}

\section{El biohuerto}

Un biohuerto es un área donde se practica la siembra, el manejo y conducción de cultivos de hortalizas con aplicación de materia orgánica. Esta producción se puede realizar a campo abierto donde se acelera su desarrollo vegetativo de las hortalizas. La producción de hortalizas en los biohuertos, son más sanas, 


\section{Journal of the Academy | 99|}

ecológicas frescas y con alto contenido de vitaminas y minerales, muy importante para la alimentación, especialmente para los niños (CEE Rafael Narváez cadenillas, 2005).

La importancia de lo biohuertos en una sociedad, ahora en la actualidad han sido dejadas de lado por países en la cual su educación ha logrado alcanzar un mejor nivel. Como efecto de la constante modernización de los países a causa de la globalización, y por ende adquisición de nuevas metodologías y tecnologías para la enseñanza, es que muchas de ellas han abandona la tradición del biohuerto, sino que han generado una serie de desigualdades, en los cuales son los países pobres los que cultivan y los modernos que consumen. Es por ello que Santana $(2013$, a) al hablar de huerto no cabe duda de que hablamos de patrimonio, pero también de valores, identidad, usos y costumbres; de jerarquías de poder; de movimientos sociales; de sostenibilidad; de alimentación; de ecología; de valores; de política; de género, etc. (pág. 3)

Esto por un lado socioeconómico, por el lado ambiental Santana $(2013, b)$ en la actualidad se ha pasado de considerar el paisaje como el marco estético de la actividad humana a considerarlo como un recurso en sí mismo. (pág., 4)

Un biohuerto es un área donde se practica la siembra, el manejo y conducción de cultivos de hortalizas con aplicación de materia orgánica. Esta producción se puede realizar a campo abierto donde se acelera su desarrollo vegetativo de las hortalizas.

La producción de hortalizas en los biohuertos, son más sanas, ecológicas frescas y con alto contenido de vitaminas y minerales, muy importante para la alimentación, especialmente para los niños. (Milachay, 2016)

\section{Biohuerto Escolares}

Los biohuertos escolares, han tomado o vienen tomando importancia y relevancia en la política educativa, son parte de la responsabilidad del docente enseñar correctamente no solo la conservación sino también la valoración que estos implican, así como de descubrir nuevas técnicas que les permita relacionar sus actividades pedagógicas con las actividades propias de lo que requiere el implementar un biohuerto. Tal como Santana $(2013$, c) refuerza lo señalado al establecer que:

“...el biohuerto escolar desempeña un papel activo como espacio de conocimiento y de construcción de la propia visión del mundo, dentro de una estrategia para conformar personas con criterio, democráticas y solidarias..." (pág., 3)

Asimismo, es de suma importancia por los diversos conocimientos que se logra obtener en estudiantes en etapa temprana de la escuela. Espinosa consideraba que:

...La agricultura ecológica consiste en la integración de los conocimientos de la agricultura tradicional con las modernas investigaciones biológicas y tecnológicas. Este es el modelo a seguir, no sólo por cuestiones de salud o respeto al medio ambiente, sino que por encima de todo hemos de hablar de cultura, esa cultura que se está perdiendo a costa de un progreso mal entendido y orientarse hacia el camino del progreso sostenible”. (Espinosa, 2010:71).

Así mismo, es importante señalar que no sólo la implementación del biohuerto genera el aprendizaje de nuevas capacidades y fortalezas en estudiantes y docentes, sino que también genera la oportunidad de cultivar nuevos alimentos que puedan ser utilizados para el consumo de los propios estudiantes, siendo esta la oportunidad también de nutrirse adecuadamente, que como se ha observado dentro de las principales problemas en Huánuco, ante la falta de diversidad agrícola en determinadas zonas de esta región. Según Escutia (2009): "Es una experiencia agrícola primordialmente educativa, alimentaria, de protección a la salud, de conservación del suelo y del trabajo comunitario". 


\section{Journal of the Academy $|100|$}

En suma, se vuelve un "aula práctica" donde el niño, desde los primeros grados de educación primaria, no solo se pone en contacto con la naturaleza, sino que interactúa con los compañeros, profesores y miembros de la comunidad, desarrollando conocimientos, valores y habilidades sobre agroecología para la conservación del medio ambiente. El biohuerto permite integrar todas las líneas de acción educativa, haciendo del proceso de aprendizaje algo vivo y natural.

La FAO (2009) propone las alternativas para implementar un biohuerto escolar:

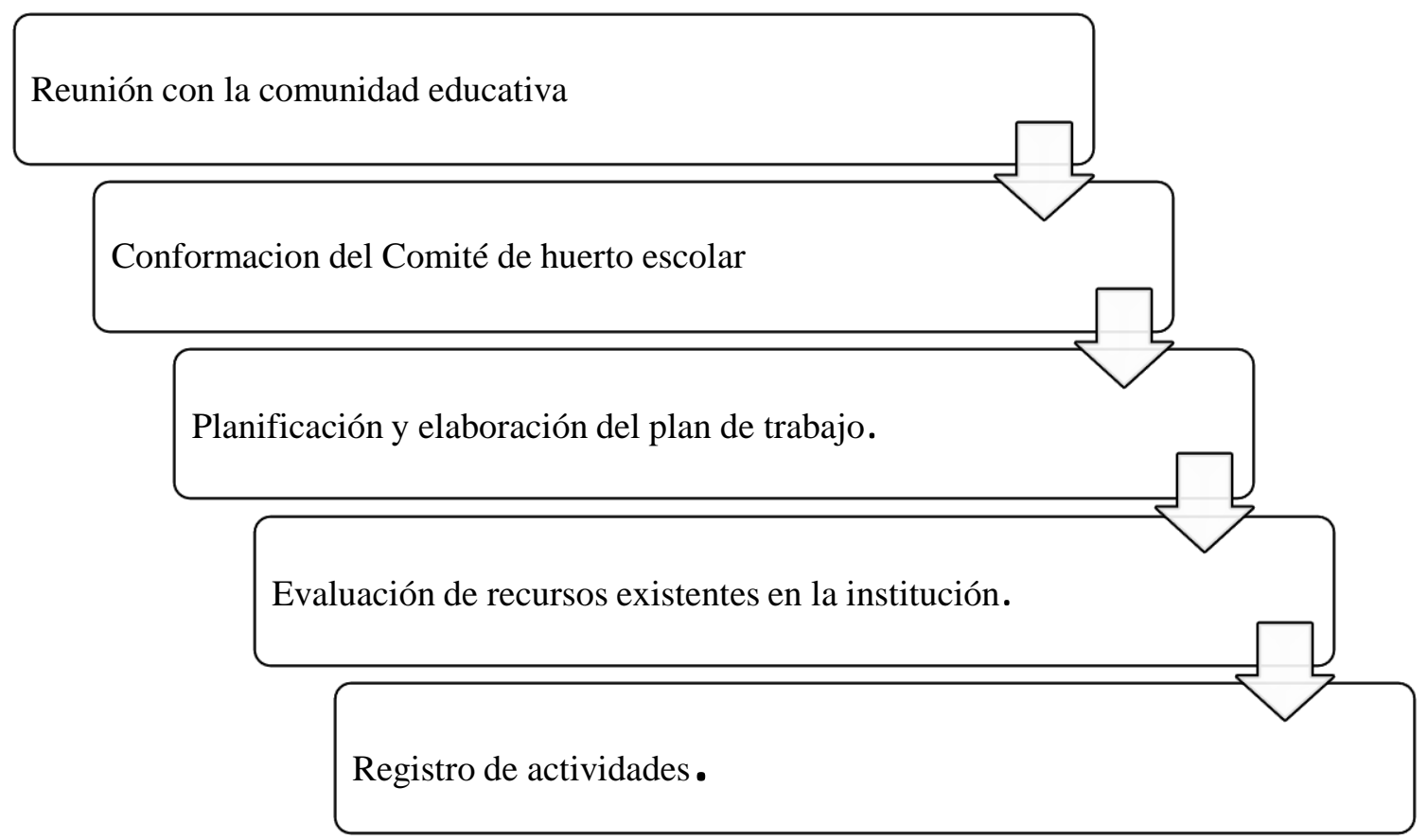

Fuente: Manual para la implementación del biohuerto escolar

FAO 2009

\section{Principios que sustentan la aplicación del biohuerto como estrategia pedagógica}

Los principios que sustentan la aplicación del biohuerto como estrategia pedagógica, según el Ministerio de Educación (2018), son:

\section{a. Principio de la construcción de los propios aprendizajes:}

- El aprendizaje como proceso de construcción interno, activo, individual e interactivo con el medio social y natural.

- Estudiantes aprenden a utiliza estructuras lógicas que dependen de variables como los aprendizajes adquiridos anteriormente y el contexto sociocultural, geográfico, lingüístico y económico productivo.

\section{b. Principio de significatividad de los aprendizajes:}

- Es posible si se relaciona los nuevos conocimientos con los que ya se poseen, y tomando en cuenta contextos, la realidad y la diversidad del estudiante.

- Deben estar interconectados con la vida real y las prácticas sociales de cada cultura.

- Se deben ofrecer experiencias que permitan aprender en forma profunda y amplia, es necesario dedicar tiempo a enseñar haciendo uso de diversas metodologías; mientras más sentidos puestos en acción, mayores conexiones se pueden establecer entre los aprendizajes previos y los nuevos. 


\section{Journal of the Academy $|101|$}

\section{c. Principio de organización de los aprendizajes:}

- Las relaciones que se establecen se amplían a través del tiempo y de la oportunidad para aplicarlos en la vida, lo que permite establecer nuevas relaciones con otros conocimientos y desarrollar la capacidad para evidenciarlas.

- Los aprendizajes se dan en los procesos pedagógicos, entendidos como interacciones en las sesiones de enseñanza - aprendizaje; en estos procesos hay que entender que tanto el docente como los estudiantes portan en sí la influencia y los condicionamientos de su salud, de su herencia, de su propia historia, de su entorno escolar, sociocultural, ecológico, ambiental y mediático; estos elementos intervienen en los procesos y en la calidad de los resultados de aprendizaje, por ello la importancia de considerarlos en la organización de los aprendizajes.

\section{d. Principio de la integralidad de los aprendizajes:}

- Deben abarcar el desarrollo integral de los estudiantes, de acuerdo con las características individuales de cada persona.

- Debe contribuir a la consolidación de las capacidades adquiridas por los estudiantes en su vida cotidiana y el desarrollo de nuevas capacidades a través de las áreas del currículo.

- Es pertinente el respeto de los ritmos individuales, estilos de aprendizaje y necesidades 28 educativas de los educandos, según sea el caso.

\section{e. Principio de evaluación de los aprendizajes:}

- La metacognición y la evaluación en sus diferentes formas, sea por el docente, estudiante u otro agente educativo, son necesarios para promover la reflexión sobre los propios procesos de enseñanza y aprendizaje.

- Los estudiantes requieren de actividades pedagógicas que les permitan reconocer sus avances y dificultades, acercarse al conocimiento de sí mismos, autoevaluarse analizando sus ritmos y estilos y aceptarse y superarse permanentemente para seguir aprendiendo de sus aciertos y errores.

\section{Aplicación del biohuerto a la enseñanza de las matemáticas.}

La implementación del biohuerto en la escuela tiene como propósito, para este trabajo, el demostrar que tiene relevancia durante la enseñanza de las matemáticas, y en el caso en particular de resolución de problemas aritméticos de enunciados verbales (PAEV) en estudiantes principalmente de la región Huánuco. Para Cuenca (2014) es importante este trabajo y lo señala

Son múltiples las ocasiones en las que tendrán que utilizar y relacionar los números y las distintas operaciones y formas de expresión y razonamiento matemático, en las tareas incluidas en el huerto escolar. Por ejemplo: Plano del terreno, cálculo de superficies, áreas y volúmenes, mediciones diversas; altura de plantas, diámetros; medidas, estimación y cálculos de magnitudes, organización de la información, gráficas y estadísticas. (pág. 30)

Como es de conocimiento, uno de los fines por los cuales se enseña matemáticas es lograr que el estudiante aprenda nuevas competencias que le permitan enfrentar la realidad y convivir con ella. Se hace necesario planear nuevas estrategias para una intervención en el proceso de enseñanza, lo que motivaría un cambio de actitud por parte del docente, que lo lleve a presentar de una manera diferente los saberes que pretende mostrar y que son sujeto de enseñanza, (Merchán, 2009).

Además, va a reflejar estrategias de experiencias innovadoras, dentro de los procesos de enseñanzaaprendizaje. Por ello es necesario manejar habilidades para la implementación de actividades donde los niños interactúen directamente con su entorno, aplicando la interdisciplinariedad como objeto principal 


\section{Journal of the Academy $|102|$}

en los procesos del saber y el saber hacer, en este sentido cobra importancia el huerto escolar en donde desarrolle sus competencias matemáticas.

Cabe señalar que este saber hacer, implica nuevas directrices en las prácticas educativas, ya que con esto se deja de lado el constante hábito de trabajar dentro del aula, para explorar y vivenciar las riquezas del entorno. Los niños pasan a ser gestores de su propio conocimiento y aprendizaje en el aprendizaje de las matemáticas realizando la resolución de problemas aritméticos de enunciados verbales.

Así también, los proyectos productivos llevados a la comunidad, son importantes debido a que los docentes tienen una mayor comunicación con la comunidad de padres involucrándolos en programas educativos de la institución educativa en estudio.

Las matemáticas es una de las áreas con más dificultad de aprendizaje por parte de los alumnos. Y al encontrarse la escuela en una zona donde predominan las actividades agrícolas y pecuarias, lo que se quiere lograr es que los estudiantes estén en un ambiente familiar donde se tenga una huerta y a partir de ella plantear diferentes problemas matemáticos.

\section{Resolución de problemas aritméticos de enunciados verbales (PAEV).}

Cantero, Hidalgo y otros (2003, pág. 9) la enseñanza de las aritméticas se debe a las siguientes razones:

- Constituyen un procedimiento para cuantificar situaciones de la vida diaria, es decir, para aplicar modelos matemáticos a situaciones concretas.

- Permiten practicar, ensayar y aplicar el lenguaje matemático como una parte del código lingüístico ordinario, ya que la clasificación de los problemas desde el punto de vista de su estructura semántica requiere hacer un análisis de la información verbal que contienen.

- Los problemas aritméticos son una vía para trascender la realidad, aplicando una forma específica de tratamiento de los datos, que hace posible volver a integrar y explicar de forma más satisfactoria esa realidad de la que había partido.

- La resolución de problemas es un medio de aprendizaje y refuerzo de contenidos.

- La resolución activa de problemas es considerada como el método más conveniente de aprender matemáticas.

- La clave de la resolución de los problemas está en el nivel de integración que el niño tiene entre el razonamiento cuantitativo y el conteo.

- La resolución de problemas requiere un alto grado de comprensión, de razonamiento y de memoria, así como la integración de destrezas cognitivas.

- La resolución de los problemas es la vertiente utilitarista e instrumental de las matemáticas. Para la mayor parte de los alumnos, éste va a ser el único contacto que en su vida futura tendrán con los conocimientos matemáticos.

Como lo ha expresado el autor, la intención de la aritmética es la resolución de problemas. Estos problemas son considerados como un determinado u obstáculo que las personas a través de diversos métodos deberán resolver; en el caso de las matemáticas que cuentan con un grado de dificultad de acuerdo al nivel académico de los alumnos, estos deberán ser evaluados por el docente para ayudar a la resolución de los mismos y evitar que se estresen o se frustren.

En ese sentido, un PAEV es un problema aritmético que se expresa o enuncia en un contexto de información verbal o gráfica. los PAEV son importantes debido a que en su proceso de solución supone un procedimiento sencillo y al alcance de los niños para lograr la modelización de situaciones de la vida diaria. Es así como éstos se convierten en el campo de entrenamiento en el que los estudiantes traducen el lenguaje ordinario a la aplicación del lenguaje matemático. (Polo, 2019, p. 105)

(Castro, De Corte, Pozo en Polo, 2015) señalan que existen dificultades separados en dos categorías: dificultades sintácticas y dificultades semánticas. 


\section{Journal of the Academy | $103 \mid$}

Figura 1: Diferencia entre dificultades

Fuente: Resolución de problemas aritméticos con enunciado verbal (PAEV) mediante el uso de Mangus Classroom en estudiantes de básica primaria de barranquilla

\begin{tabular}{|c|c|}
\hline SINTÁCTICAS & SEMÁNTICAS \\
\hline $\begin{array}{l}\text { la forma en que viene enunciado el problema. } \\
\text { Entre estas tenemos las siguientes: Formato de } \\
\text { presentación del problema; expresiones } \\
\text { verbales; representaciones; expresiones } \\
\text { simbólicas; longitud del enunciado; posición } \\
\text { de la pregunta en el enunciado; presencia o no } \\
\text { de datos en la pregunta; datos numéricos; } \\
\text { tamaño de los números; tipo de número; orden } \\
\text { de los datos; datos superfluos; contexto de la } \\
\text { información; situación real; situación ficticia } \\
\text { y estilo de redacción. }\end{array}$ & $\begin{array}{l}\text { Las dificultades semánticas están } \\
\text { relacionadas, en general, con los significados } \\
\text { de las distintas expresiones en el enunciado y } \\
\text { su relación con los conceptos y } \\
\text { procedimientos propios de la matemática. } \\
\text { Entre otras tenemos las siguientes: Estructura } \\
\text { de la pregunta; combinación (Relación } \\
\text { estática entre los datos); cambio (Relación } \\
\text { dinámica entre los datos); comparación } \\
\text { (Cuánto más, más qué, menos que...) e } \\
\text { igualación (Tantos como...cuánto falta } \\
\text { para...) }\end{array}$ \\
\hline
\end{tabular}

De La Cruz (2017) señala que:

los estudiantes al resolver problemas matemáticos aprenden a pensar matemáticamente, que es un proceso complejo y dinámico que resulta de la acción recíproca de varios factores como los socioculturales, cognitivos, y afectivos así mismo desarrollan capacidades y actitudes determinando hechos, estableciendo relaciones, potenciando su autonomía, su razonamiento, su creatividad, su imaginación, promoviendo la utilidad de los materiales concretos; estimulando el trabajo cooperativo, la toma de decisiones para alcanzar un aprendizaje con alto nivel de significatividad vinculados con las prácticas sociales y culturales "a través de", "sobre" y "para" la resolución de problemas en diversos contextos.

Es por ello que la autora en su investigación propone que este estilo de aprendizaje se puede sustentar en las siguientes teorías:

\section{Teoría Psico genética de Jean Piaget}

El conocimiento matemático es el resultado del desarrollo interno del sujeto, producto de un proceso individual de reflexión e interiorización partiendo de la interactuación con los objetos. El sujeto que accede a las operaciones formales es capaz de resolver cualquier situación problemática planteada, independientemente de qué contenido tenga. Lo importante desde esta visión no es de enseñar distintos contenidos, sino la principal función del docente facilitador es de ayudar a desarrollar operaciones cognitivas básicas.

Desde el punto de vista didáctico esta teoría está aplicada a propuestas curriculares concretas que es propio de las matemáticas, donde los niños construyen su mente por abstracción reflexiva a través de las relaciones con los soportes concretos: materiales estructurados y no estructurados, las manipula y las aplica para representar cualquier situación. El proceso de aprendizaje de la matemática se da por etapas vivenciales, de manipulación, representación gráfico simbólico y la abstracción; una vez adquirido el conocimiento es procesado y no se olvida ya que es la propia experiencia de una acción.

\section{Teoría de Jerome S. Bruner Bruner}

Sustenta que el conocimiento se construye por una interacción constante con el medio cultural y social a través del cual induce al sujeto a una participación activa en el proceso de aprendizaje. El aprendizaje se presenta en su contexto cotidiano desafiando la inteligencia del sujeto e impulsándolo a resolver 25 problemas y a lograr la transferencia de lo aprendido. Se conoce el mundo de manera progresiva en tres etapas de maduración o de desarrollo intelectual por las cuales pasa el individuo, que son los modos 


\section{Journal of the Academy $|104|$}

psicológicos de conocer: modo inactivo, modo icónico y modo simbólico, que se corresponden con las etapas del desarrollo en las cuales se pasa primero por la acción, luego por la imagen y finalmente por el lenguaje. Estas etapas son acumulativas, de tal forma que cada etapa que es superada perdura toda la vida como forma de aprendizaje. Para Bruner el niño desarrolla su inteligencia poco a poco en un sistema de evolución, primero desde los aspectos más simples del aprendizaje para poder llegar hasta los más complejos. Lo importante para Bruner en la enseñanza de conceptos básicos es que se debe enseñar a los niños a pasar de manera progresiva, de un pensamiento concreto a un estadio de representación conceptual y simbólica que es el más adecuado con el crecimiento de su pensamiento; es decir en el análisis que realizan sobre el tipo de representación.

\section{Teoría social cultural de Lev Vygotsky}

Esta teoría esta basa en el aprendizaje sociocultural de cada individuo, es el medio donde se desarrolla de forma social y cultural, en este modelo de aprendizaje sociocultural sostenido por Vygotsky el desarrollo y el aprendizaje interactúan entre sí, en el cual considera al aprendizaje como un factor de desarrollo; asimismo concibe al sujeto como un ser eminentemente social. Para Vygotsky el aprendizaje escolar se produce de manera más fácil en situaciones colectivas y debe de ser congruente de acuerdo con el nivel de desarrollo del niño porque es aquí donde desarrolla su propio papel activo. Vygotsky destacó el valor de la cultura y el contexto social, que veía crecer el niño a la hora de hacerles de guía y ayudarles en el proceso de aprendizaje. Para Vygotsky el niño tiene la necesidad de actuar de manera eficaz y con independencia y tiene la capacidad para desarrollar un estado mental de 26 funcionamiento superior cuando interacciona con la cultura y con otras personas. El niño tiene un papel activo en el proceso de aprendizaje, pero no actúa solo. El andamiaje, implica guiar a través de consejos, preguntas y material que dirigen al niño mientras resuelve problemas. Pero dirigir no quiere decir explicar. Los maestros prepararan el terreno para que los estudiantes identifiquen lo que necesitan hacer, en lugar de explicarles los pasos a seguir, como sí se tratara de un algoritmo. Los estudiantes han de aprender de qué manera puede solucionar los problemas y superar obstáculos, aparte de aprender a solucionar los problemas en sí. Y todavía más importante, han de aprender a sentirse seguros con el sistema empírico. Estas teorías cognitivas producen impacto y aporta conocimiento sobre el desarrollo psicológico cognitivo en el aprendizaje del ser humano, porque el ser humano aprende a lo largo de toda su vida utilizando los procesos cognitivos para desenvolverse y enfrentarse a los retos de la sociedad actual que requiere de personas capaces y competentes dentro del campo de la matemática.

\section{Región Huánuco}

La región de Huánuco se encuentra en la parte central del Perú con un basto territorio de $37200 \mathrm{~km}^{2}$, se ubica entre la zona sierra (60\%) y la zona de ceja de selva (40\%) por lo que su territorio es rico y diverso en climas y vegetación.

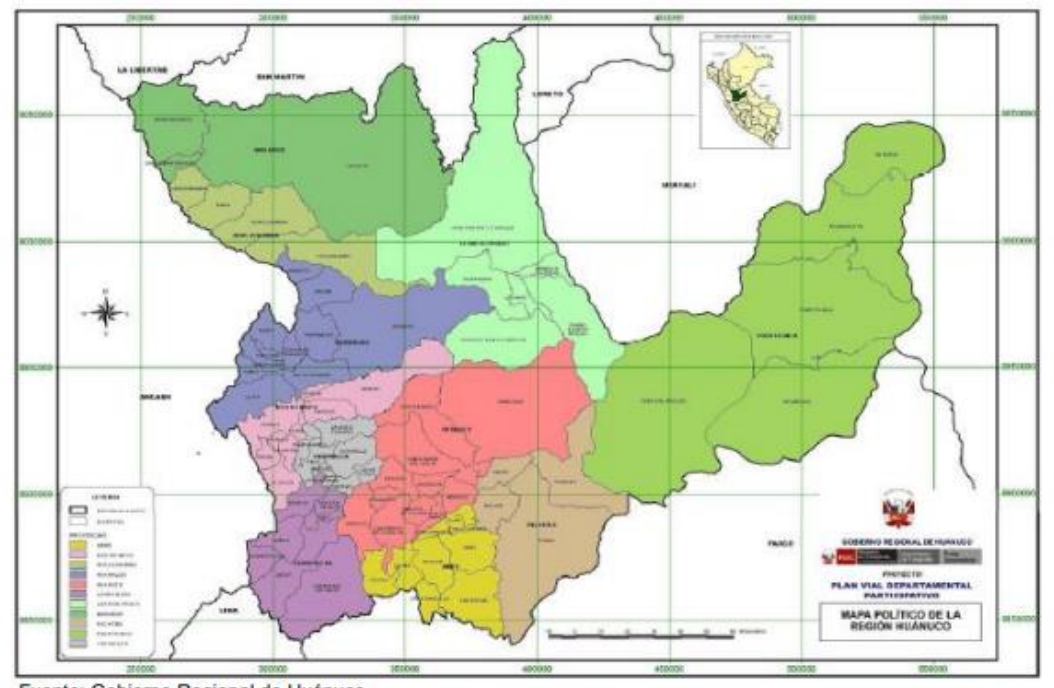

de Huánuco.

Nota: Para más detalles: http://maps.google.com/?ie=UTF8\&.1=-9.882275, $-76.26709 \&$ spn=4.696045,8.327637\&z=7 


\section{Journal of the Academy $|105|$}

En base al Censo de Población y Vivienda 2017, de los 721047 habitantes censados en Huánuco, el 41 por ciento se encontró en la provincia de Huánuco, seguido de Leoncio Prado (18 por ciento), Huamalíes (7 por ciento) y Ambo (7 por ciento), entre los principales. En el contexto nacional, Huánuco es el décimo departamento con mayor población, concentrando Lima el 35,7 por ciento y Madre de Dios, la menor $(0,5$ por ciento).

\begin{tabular}{|l|l|l|l|}
\hline Provincia & $\mathbf{N}^{\circ}$ de distritos & Superficie & Población \\
\hline Huánuco & 13 & 3592 & 293397 \\
\hline Leoncio Prado & 10 & 4943 & 127793 \\
\hline Huamalíes & 11 & 3145 & 52039 \\
\hline Ambo & 8 & 1575 & 50880 \\
\hline Pachitea & 4 & 3069 & 49159 \\
\hline Dos de Mayo & 9 & 1468 & 33258 \\
\hline Puerto Inca & 5 & 10341 & 32538 \\
\hline Marañon & 5 & 4801 & 26622 \\
\hline Yarowilca & 8 & 727 & 19897 \\
\hline Lauricocha & 7 & 1860 & 18913 \\
\hline Huacaybamba & 4 & 1744 & 1744 \\
\hline
\end{tabular}

Superficie y población

Elaboración propia

Fuente: INEI, Censo de Población y Vivienda 2017

\section{Proyecto educativo regional Huánuco 2018-2028}

Tomando en cuenta la necesidad de mejorar la calidad de la educación de su región se ha presentado el Proyecto Educativo Regional Huánuco 2018-2028 con la finalidad de contar con una educación inclusiva, intercultural, globalizada en una sociedad que poco a poco va mejorando.

El proceso de construcción del PER, ha logrado a través de diversas mesas de trabajo, simposios, panel foro, grupos focales, entrevistas y demás, junto con la participación de instituciones públicas y privadas de todos los sectores.

Este proyecto tiene como finalidad ser una guía de orientación de las políticas educativas de la región, respetando la diversidad de la población huanuqueña. Este proyecto se ha sintetizado en seis objetivos estratégicos. En ellos, se aspira a una educación en donde:

1. Todos los huanuqueños, sin excepción, logren aprendizajes de calidad. Desde esa perspectiva, es prioridad para esta región fortalecer la atención de la primera infancia y la educación básica regular y técnico productivo, garantizando con ello una educación para la vida y que responda a los retos de la globalización.

2. Instituciones inteligentes y acogedoras garantizando la formación integral de los estudiantes y, para coadyuvar en esta labor, se propone la creación del Instituto Regional de Evaluación de Calidad Educativa.

3. Contar con maestros capacitados y que ejerzan profesional y éticamente la docencia, por lo que se propone realizar un sistema de formación docente con la intención de garantizar su funcionamiento eficiente, en la cual se forma un centro de formación de directivos que garanticen una educación de alta calidad.

4. Contar con una gestión educativa estratégica y descentralizada que desarrolle el talento humano, que prepare a los niños a responder a los retos educativos de la globalización, pero también con un alto compromiso con su comunidad. 


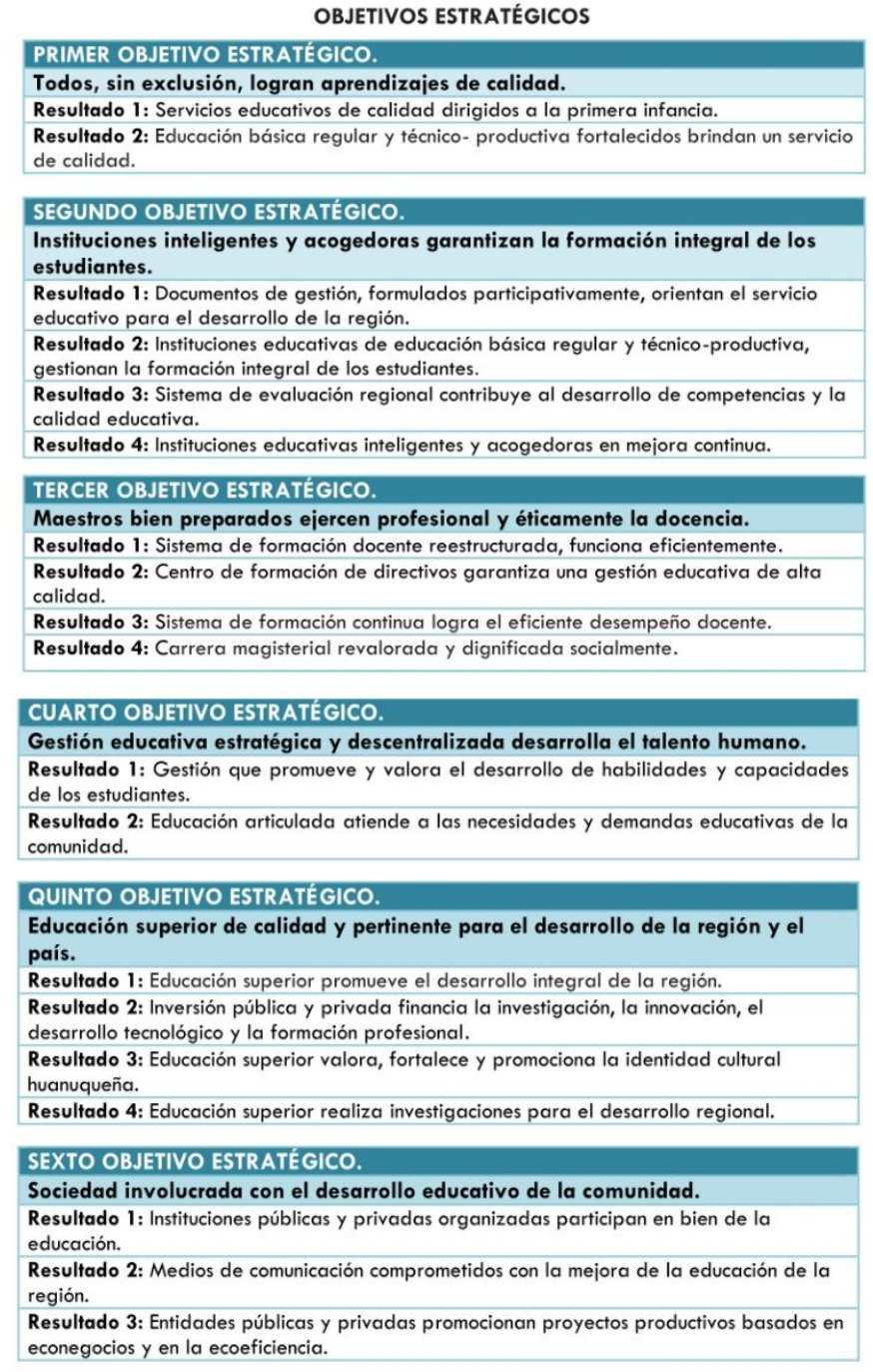

Fuente: Gobierno Regional de Huánuco Objetivos del Plan Regional de Educación

\section{CONCLUSIONES}

La aplicación de estrategias en matemáticas es necesaria para un país que cuenta con un nivel educativo muy bajo, es por ello que resulta importante que como parte de las actividades de los docentes se debe implementar biohuertos escolares en los colegios en la región Huánuco. Esta importancia no solo se enfoca en las nuevas actividades y estilos de relación que los estudiantes y docentes pueden alcanzar, sino también el cultivo de nuevas vegetaciones que permitirán una mejora alimenticia de los escolares.

Los PAEV en relación con los biohuertos no solo alcanzan la capacidad del estudiante de resolver estos problemas sino también mejorar su capacidad de retención por las verduras con una mayor variedad de vitaminas y minerales que puedan obtener.

Asimismo, las autoridades de la región Huánuco han tomado acciones no solo para mejorar la educación sino también rescatando los principales valores y costumbres de la región Huánuco para una educación inclusiva. 


\section{Journal of the Academy | $107 \mid$}

\section{REFERENCIAS BIBLIOGRÁFICAS}

Avalos, P., (2019) Relación entre la estandarización y los proyectos de infraestructura de educación en Huánuco. Universidad Tecnología del Perú.

Cantero, A., Hidalgo, A., Merazyo, B., et al (2003) Resolución de problemas aritméticos en educación primaria. Recuperado de http://lvl.educarex.es/conoceryaplicarlvlylvm/F9_Resolucion_problemas_aritmeticos.pdf

CEE R. NARVÁEZ CADENILLAS. (2005). El Biohuerto Escolar: Un proyecto ecopedagógico de producción. Trujillo: Centro Educativo Experimental Rafael Narváez Cadenillas. Proyectos, Experimentos e Investigaciones. Investigaciones en el Área de Ecología

Cuenca, G., (2014). El huerto como laboratorio de matemáticas: Aprendizaje de los números racionales positivos (Tesis de Maestría). Universidad Nacional de Colombia

De La Cruz, O., (2017). Programa "La Cajita Mágica" en resolución de problemas aritméticos de enunciado verbal en estudiantes de una Institución Estatal de Ate, 2016. (Tesis de Maestría). Universidad César Vallejo. Lima, Perú.

Escutia, M. (2009). El huerto escolar ecológico. Editorial GRAO. España

Espinosa, J. M. (2010). Elaboración de un huerto ecológico: inicio a la educación Ambiental, en Revista digital Transversalidad Educativa núm. 30, pp. 70-83. Editorial enfoques educativos, S.L

Merchán, F. (2013). La cuestión del cambio de la práctica de la enseñanza y la necesidad de una teoría de la acción en el aula. Recuperado de http://www.rieoei.org/deloslectores/2679Iglesias.pdf

Marchán, M., Valverde, F., Novoa, V., y Pumisacho, M. (2009). Guía para facilitar el aprendizaje en el manejo integrado de suelos en el cultivo de la papa. Instituto Nacional Autónomo de Investigaciones Agropecuarias (INIAP). Ecuador

Milachay, R., (2016) Estilos de aprendizaje, liderazgo democrático en la resolución de problemas en estudiantes de los CEBAS. Tesis de Doctorado. Universidad César Vallejo. Lima, Perú

Ministerio de Educación. (2015). Rutas de aprendizaje de matemática. Lima, Perú: Quad/Graphics.

Ministerio de Educación del Perú. (2018). Buenas prácticas docentes. Recuperado de http://www.minedu.gob.pe/buenaspracticasdocentes/

Polo, H., (2019). Resolución de Problemas Aritméticos con Enunciado Verbal (PAEV) mediante el uso de Mangus Classroom en estudiantes de básica primaria de barranquilla (Tesis de Maestría). Universidad de la Costa. Colombia

República del Perú, (2018), Evaluación Censal de Estudiantes. Instituto Nacional de Estadística e Informática. Recuperado de www.inei.gob.pe

Rojas, A., Salinas, L. y Ch, J. (2019). Clima institucional y gestión pedagógica en educación secundaria, Huánuco. 2017. Revista Opcion Vol. 35, 90: 83-99

Santana, D., (2013) El biohuerto escolar como recurso en la educación intercultural (Trabajo de grado). Facultat de Magisteri, Universitat de Valencia. 